\title{
Some More Results on Harmonic Mean Graphs
}

\author{
S. S. Sandhya (Corresponding author) \\ Department of Mathematics, Sree Ayyappa College for Women \\ Chunkankadai, Kanyakumari 629 807, Tamilnadu, India \\ E-mail: sssandhya2009@gmail.com
}

S. Somasundaram

Department of Mathematics, Manonmaniam Sundaranar University

Tirunelveli 627 012, Tamilnadu, India

E-mail: somumsu@rediffmail.com

R. Ponraj

Department of Mathematics, Sri Paramakalyani College

Alwarkurichi 627 412, Tamilnadu, India

E-mail: ponrajmath@gmail.com

Received: November 10, 2011 Accepted: November 28, 2011 Published: February 1, 2012

doi:10.5539/jmr.v4n1p21 URL: http://dx.doi.org/10.5539/jmr.v4n1p21

\begin{abstract}
A Graph $G=(V, E)$ with $p$ vertices and $q$ edges is called a harmonic mean graph if it is possible to label the vertices $x \in V$ with distinct labels $f(x)$ from $1,2, \ldots, q+1$ in such a way that when each edge $e=u v$ is labeled with $f(u v)=\left\lceil\frac{2 f(u) f(v)}{f(u)+f(v)}\right\rceil$ or $\left\lfloor\frac{2 f(u) f(v)}{f(u)+f(v)}\right\rfloor$ then the edge labels are distinct. In this case $f$ is called Harmonic mean labeling of $G$.

The concept of Harmonic mean labeling was introduced in (Somasundaram, Ponraj \& Sandhya). In (Somasundaram, Ponraj \& Sandhya) and (Sandhya, Somasundaram \& Ponraj, 2012) we investigate the harmonic mean labeling of several standard graphs such as path, cycle comb, ladder, Triangular snakes, Quadrilateral snakes etc. In the present paper, we investigate the harmonic mean labeling for a polygonal chain, square of the path and dragon. Also we enumerate all harmonic mean graph of order $\leq 5$.
\end{abstract}

Keywords: Graph, Harmonic mean graph, Polygonal chain, Square of a path, Dragon

AMS subject classification: 05C 78

\section{Introduction}

The graph considered here will be finite, undirected and simple. Terms not defined here are used in the sense of Harary (Harary, 1988). The symbols $V(G)$ and $E(G)$ will denote the vertex set and edge set of a graph $G$. The Cardinality of the graph $G$ is called the order of $G$. The Cardinality of its edge set is called the size of $G$. The graph $G-e$ is obtained from $G$ by deleting an edge $e$. The sum $G_{1}+G_{2}$ of two graphs $G_{1}$ and $G_{2}$ has vertex set $V\left(G_{1}\right) \cup V\left(G_{2}\right)$ and edge set $E\left(G_{1}+G_{2}\right)=E\left(G_{1}\right) \cup E\left(G_{2}\right) \cup\left\{u v: u \in V\left(G_{1}\right)\right.$ and $\left.v \in V\left(G_{2}\right)\right\}$. The Union of two graphs $G_{1}$ and $G_{2}$ is a graph $G_{1} \cup G_{2}$ with vertex set $V\left(G_{1} \cup G_{2}\right)=V\left(G_{1}\right) \cup V\left(G_{2}\right)$ and $E\left(G_{1} \cup G_{2}\right)=E\left(G_{1}\right) \cup E\left(G_{2}\right)$. The Square $G^{2}$ of the graph $G$ has $V\left(G^{2}\right)$ with $u, v$ adjacent in $G^{2}$ whenever $d(u, v) \leq 2$ in the graph $G$. A dragon is a graph formed by joining an end vertex of path $P_{m}$ to the vertex of the cycle $C_{n}$. It is denoted $C_{n} @ P_{m}$. The graph $C_{n} \hat{o} K_{1, m}$ is obtained $C_{n}$ and $K_{1, m}$ by identifying any vertex of $C_{n}$ and the central vertex of $K_{1, m}$.

S. Somasundaram and R. Ponraj introduced mean labeling of graphs in (Somasundaram \& Ponraj, 2003a) and investigate mean labeling for some standard graphs in (Somasundaram \& Ponraj, 2003b) and in (Somasundaram \& Ponraj, 2004). We introduce Harmonic mean labeling of graphs in (Somasundaram, Ponraj \& Sandhya) and proved Harmonic mean labeling of some standard graphs in (Sandhya, Somasundaram \& Ponraj, 2012). In this paper we prove that polygonal chains, square of a path and dragons are harmonic mean graphs. Finally we investigate all harmonic mean graph of order $\leq 5$. 
We shall make frequent reference to the following results.

Theorem 1.1 (Somasundaram, Ponraj \& Sandhya) Any path is a harmonic mean graph.

Theorem 1.2 (Somasundaram, Ponraj \& Sandhya) Any cycle $C_{n}, n \geq 3$ is a harmonic mean graph.

Theorem 1.3 (Somasundaram, Ponraj \& Sandhya) The complete graph the $K_{n}$ is a harmonic graph if and only if $n \leq 3$.

Theorem 1.4 (Somasundaram, Ponraj \& Sandhya) The complete bipartite graph $K_{1, n}$ is a harmonic mean graph if and only if $n \leq 7$.

Theorem 1.5 (Sandhya, Somasundaram \& Ponraj, 2012) Any wheel $W_{n}$ is not a harmonic mean graph.

Theorem 1.6 (Sandhya, Somasundaram \& Ponraj, 2012) $C_{m} \cup P_{n}, n>1, C_{m} \cup C_{n}, m, n \geq 3$ is a harmonic mean graph.

Theorem 1.7 (Sandhya, Somasundaram \& Ponraj, 2012) $K_{n}-e, n>4$ is not a harmonic mean graph.

Remark 1.8 (Sandhya, Somasundaram \& Ponraj, 2012) If $p>q+1$, then the graph $G=(p, q)$ is not a harmonic mean graph.

Remark 1.9 (Sandhya, Somasundaram \& Ponraj, 2012) If $n \leq 4, K_{n}-e$ is a harmonic mean graph.

\section{Harmonic Mean Labeling}

Definition 2.1 A Graph $G$ with $p$ vertices and $q$ edges is called a harmonic mean graph if it is possible to label the vertices $x \in V$ with distinct labels $f(x)$ from $1,2, \ldots, q+1$ in such a way that when each edge $e=u v$ is labeled with $f(u v)=\left\lceil\frac{2 f(u) f(v)}{f(u)+f(v)}\right\rceil$ or $\left\lfloor\frac{2 f(u) f(v)}{f(u)+f(v)}\right\rfloor$ then the edge labels are distinct. In this case $f$ is called Harmonic mean labeling of $G$.

Definition 2.2 A Polygonal chain $G_{m, n}$ is a connected graph all of whose $m$ blocks are polygons $\left(C_{n}\right)$.

Theorem 2.3 Polygonal chain $G_{m, n}$ are Harmonic mean graphs for all $m$ and $n$.

Proof: In $G_{m, n}$ chain, let $u_{1} u_{2} u_{4} u_{6} \ldots u_{n-4} u_{n-2} u_{n+1} u_{n-1} u_{n-3} u_{n-5} \ldots u_{7} u_{5} u_{3} u_{1}$ be the first cycle. The second cycle is connected to the first cycle at the vertex $u_{n+1}$. Let $u_{n+1} u_{n+2} u_{n+4} \ldots u_{2 n+1} u_{2 n-1} u_{2 n-3} \ldots u_{n+7} u_{n+5} u_{n+3} u_{n+1}$ be the second cycle. The third chain is connected to the second cycle at the vertex $u_{2 n+1}$. Let the third cycle be $u_{2 n+1} u_{2 n+2} u_{2 n+4} \ldots u_{3 n+1} u_{3 n-1} u_{3 n-3} \ldots u_{2 n+5} u_{2 n+3} u_{2 n+1}$. In general the $r^{\text {th }}$ cycle is connected to the $(r-1)^{t h}$ cycle at the vertex $u_{r n+1}$. Let the $r^{t h}$ cycle be $u_{r n+1} u_{r n+2} u_{r n+4} u_{r n+6} \ldots u_{(r+1) n-4} u_{(r+1) n-2} u_{(r+1) n+1} u_{(r+1) n-1} u_{(r+1) n-3} u_{(r+1) n-5} u_{(r+1) n-7} \ldots u_{r n+5} u_{r n+3} u_{r n+1}$.

Magnified figure of the $r^{\text {th }}$ cycle is given in Figure 1.

Assume the graph has $m$ cycles. Define a function $f: V\left(G_{m, n}\right) \rightarrow\{1,2, \ldots, q+1\}$ by $f\left(v_{i}\right)=i, 1 \leq i \leq m n-1, f\left(v_{n}\right)=$ $m n+1$. Then the label of the edge is given below

$$
\begin{gathered}
f\left(u_{m n+1} u_{m n+2}\right)=m n+1 \\
f\left(u_{m n+i} u_{m n+i+2}\right)=m n+i+1 \\
f\left(u_{(m+1) n-2} u_{(m+1) n+1}\right)=(m+1) n-1 \\
f\left(u_{(m+1) n+1} u_{(m+1) n-1}\right)=(m+1) n
\end{gathered}
$$

Since the graph $G_{m, n}$ has distinct edge labels, $G_{m, n}$ is a harmonic mean graph.

Example 2.4 Harmonic mean labeling of $G_{4,9}$ chain is in Figure 2.

Now we investigate the square of a path.

Theorem 2.5 The graph $P_{n}^{2}$ is a harmonic mean graph.

Proof: Let $P_{n}$ be the path $u_{1}, u_{2}, \ldots, u_{n}$. Clearly $P_{n}^{2}$ has $n$ vertices and $2 n-3$ edges. Define $f: V\left(P_{n}^{2}\right) \rightarrow\{1,2, \ldots, q+1\}$ by $f\left(u_{i}\right)=2 i-1,1 \leq i \leq n-1$ and $f\left(u_{n}\right)=2 n-2$. The label of the edge $u_{i} u_{i+1}$ is $2 i-1,1 \leq i \leq n-1$. The label of the edge $u_{i} u_{i+2}$ is $2 i, 1 \leq i \leq n-2$. The label of the edge $u_{n-1} u_{n}$ is $2 n-3$. Hence $P_{n}^{2}$ is a harmonic mean graph.

Example 2.6 Harmonic mean labeling of $P_{8}^{2}$ is given in Figure 3.

Theorem 2.7 Dragon's $C_{n} @ P_{m}$ are harmonic mean graphs.

Proof: Let $u_{1} u_{2} \ldots u_{n}$ be the cycle $C_{n}$ and $v_{1} v_{2} \ldots v_{m}$ be the path $P_{m}$. Identify $u_{n-1}$ with $v_{1}$. Define a function $f$ : $V\left(C_{n} @ P_{m}\right) \rightarrow\{1,2, \ldots, q+1\}$ by $f\left(u_{i}\right)=i, 1 \leq i \leq n-3, f\left(u_{n-2}\right)=n-1, f\left(u_{n-1}\right)=n, f\left(u_{n}\right)=n-2$ and $f\left(v_{i+1}\right)=$ $n+i, 1 \leq i \leq m-1$. Clearly $f$ is a harmonic mean labeling

Example 2.8 A harmonic mean labeling of $C_{6} @ P_{7}$ is given in Figure 4. 
Theorem 2.9 The graph $C_{n} \hat{o} K_{1,1}$ is a harmonic mean graph.

Proof: We know that, $C_{n} \hat{o} K_{1,1}=C_{n} @ P_{2}$. Hence the proof follows from the Theorem 2.7.

Remark 2.10 The graph $C_{n} \hat{o} K_{1,2}$ is also a harmonic mean graph.

Theorem 2.11 Let $C_{n}$ be the cycle $u_{1} u_{2} u_{3} \ldots u_{n}$. Let $G$ be the graph with $V(G)=V\left(C_{n}\right) \cup\left\{v_{1}, v_{2}\right\}$ and $E(G)=E\left(C_{n}\right) \cup$ $\left\{u_{1} v_{1}, u_{n} v_{2}\right\}$. Then $G$ is a harmonic mean graph.

Proof: Define a function $f: V(G) \rightarrow\{1,2, \ldots, q+1\}$ by $f\left(u_{i}\right)=i+1,1 \leq i \leq n, f\left(v_{1}\right)=1$ and $f\left(v_{2}\right)=n+2$. Then we get distinct edge labels from $1,2, \ldots, q$. Obviously $f$ is a harmonic mean labeling for $G$.

Example 2.12 The harmonic mean labeling of $\mathrm{G}$ is given in Figure 5.

Theorem 2.13 Let $G$ be the graph obtained from $K_{4}-e$ by attaching $P_{n}$ with the vertex of degree 3 in $K_{4}-e$. Then $G$ is a harmonic mean graph.

Proof: Let $v_{1}, v_{2}, v_{3}$ and $v_{4}$ be the vertices of $G=K_{4}-e$ and $e=v_{1} v_{3}$. Let $P_{n}$ be the path $u_{1} u_{2} u_{3} \ldots u_{n}$. Identify $u_{1}$ with $v_{4}$. Define $f: V(G) \rightarrow\{1,2, \ldots, q+1\}$ by $f\left(v_{1}\right)=1, f\left(v_{2}\right)=3, f\left(v_{3}\right)=4 f\left(v_{4}\right)=6, f\left(u_{i+1}\right)=6+i, 1 \leq i \leq n-1$. Obviously $G$ is a harmonic mean graph.

Example 2.14 Harmonic mean labeling of $G$ is given in Figure 6.

Next we have

Theorem 2.15 Let $G$ be the graph obtained from $K_{4}-e$ by attaching $P_{n}$ to the vertex of the degree 2 in the graph $K_{4}-e$. Then $G$ is a harmonic mean graph.

Proof: Let $v_{1}, v_{2}, v_{3}$ and $v_{4}$ be the vertices of $G=K_{4}-e$ and $u_{1} u_{2} u_{3} \ldots u_{n}$ be the path $P_{n}$. Define $f: V(G) \rightarrow\{1,2, \ldots, q+1\}$ by $f\left(v_{1}\right)=1, f\left(v_{2}\right)=3, f\left(v_{3}\right)=5 f\left(v_{4}\right)=7, f\left(u_{i+1}\right)=5+i, 1 \leq i \leq n-1$. Clearly $\mathrm{f}$ is a harmonic mean labeling.

Example 2.16 Harmonic mean labeling of $G$ is given in Figure 7.

\section{Harmonic Mean Graph of Order $\leq 5$}

Theorem 3.1 The following graphs of Order $\leq 5$ are harmonic mean graphs

i) $P_{1}, P_{2}, P_{3}, P_{4}, P_{5}$

ii) $C_{3}, C_{4}, C_{5}$

iii)
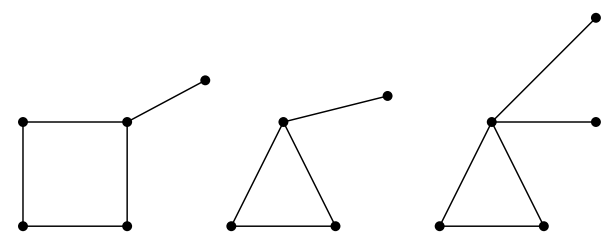

iv) $K_{1,3}, K_{1,4}$

v) $C_{3} \cup P_{2}$

vi)

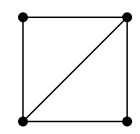

Proof: Since all paths are harmonic mean graphs, the graphs in case (i) are harmonic mean graphs by Theorem 1.1 The graphs in case (ii) are harmonic mean graphs by Theorem 1.2 The graph in case (iii) $C_{3} \hat{o} K_{1,1}, C_{3} \hat{o} K_{1,2}$ and $C_{4} \hat{o} K_{1,1}$ are harmonic mean graphs by Theorem 2.9 and Remark 2.10. The graphs in case (iv) are harmonic mean graphs by Theorem 1.4. The graphs in case (v) is a harmonic mean graph by Theorem 1.6. The graph in case (vi) are harmonic mean graphs by Remark 1.9 .

Theorem 3.2 The following graphs of order $\leq 5$ are not harmonic mean graphs.

i) $K_{2}{ }^{c}, K_{3}{ }^{c}, K_{4}{ }^{c}, K_{5}{ }^{c}$, 

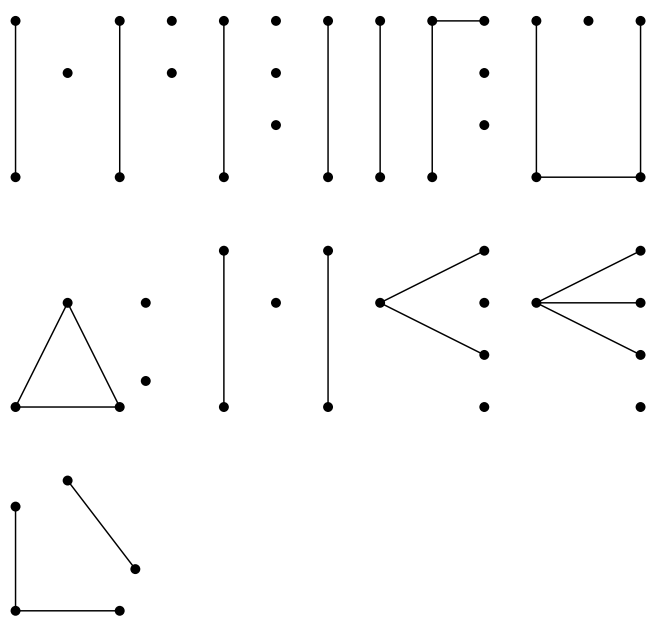

ii) $K_{4}, K_{5}, K_{4} \cup K_{1}$

iii) $W_{4}, W_{5}$

iv) $K_{5}-e$

Proof: The graphs in case (i) are not harmonic mean graph by Remark 1.8 The graphs in case (ii) are not harmonic mean graphs by Theorem 1.3. The graphs in case (iii) are not harmonic mean graph by Theorem 1.5. The graph in case (iv) are not harmonic mean graph by Theorem 1.7 .

Remark 3.3 The remaining 16 graphs of order $\leq 5$ are given in Table 3.1 and Table 3.2. They are shown to be harmonic mean graphs by giving a specific harmonic mean labeling.

The Table 3.3 shows the number of graphs of order $\leq 5$ which are harmonic mean and which are not harmonic mean.

\section{References}

Gallian, J. A. (2010). A dynamic survey of graph labeling. The Electronic Journal of Combinatorics, 17, \#DS6.

Harary, F. (1988). Graph Theory. Narosa publishing House Reading New Delhi.

Sandhya, S. S., Somasundaram, S., \& Ponraj, R. (2012). Some Results on harmonic mean graphs. to appear in International journal of contemporary Mathematical sciences, 7, 4.

Somasundaram, S., \& Ponraj, R. (2003a). Mean Labelings of graphs. National Academy science Letters, 26, 210-213.

Somasundaram, S., \& Ponraj, R. (2003b). Some Results on Mean graphs. Pure and applied Mathematika sciences, 58, 29-35.

Somasundaram, S., \& Ponraj, R. (2003c). Non-existence of mean labeling for a wheel. Bulletin of pure and Applied sciences, 22E, 1, 103-111.

Somasundaram, S., \& Ponraj, R. (2004). On mean graphs of order $\leq 5$. Journal of Decision and mathematical sciences, 9,1-3.

Somasundaram, S., Ponraj, R., \& Sandhya, S. S. Harmonic mean labelings of graphs. communicated to Journal of Combinatorial Mathematics and Combinatorial Computing .

Somasundaram, S., \& Sandhya, S. S. (2011a). Skolem harmonic mean labelings of graphs, to appear in Bulletin of pure and applied sciences, 30E, 2.

Somasundaram, S., \& Sandhya, S. S. (2011b). Some Results on Skolem harmonic mean graphs. International journal of Mathematic Research , 3, 6, 619-625. 
Table 3.1

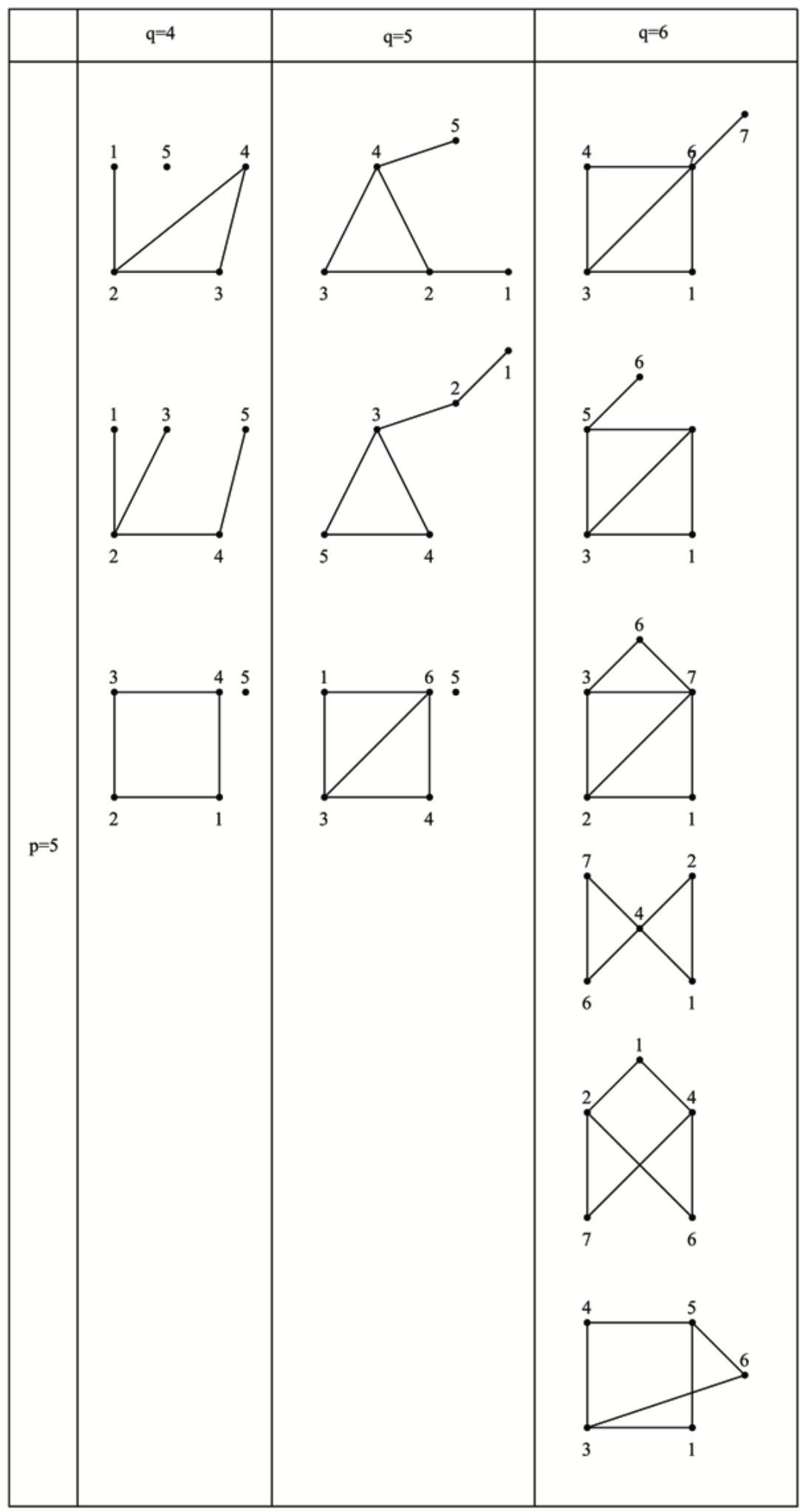


Table 3.2

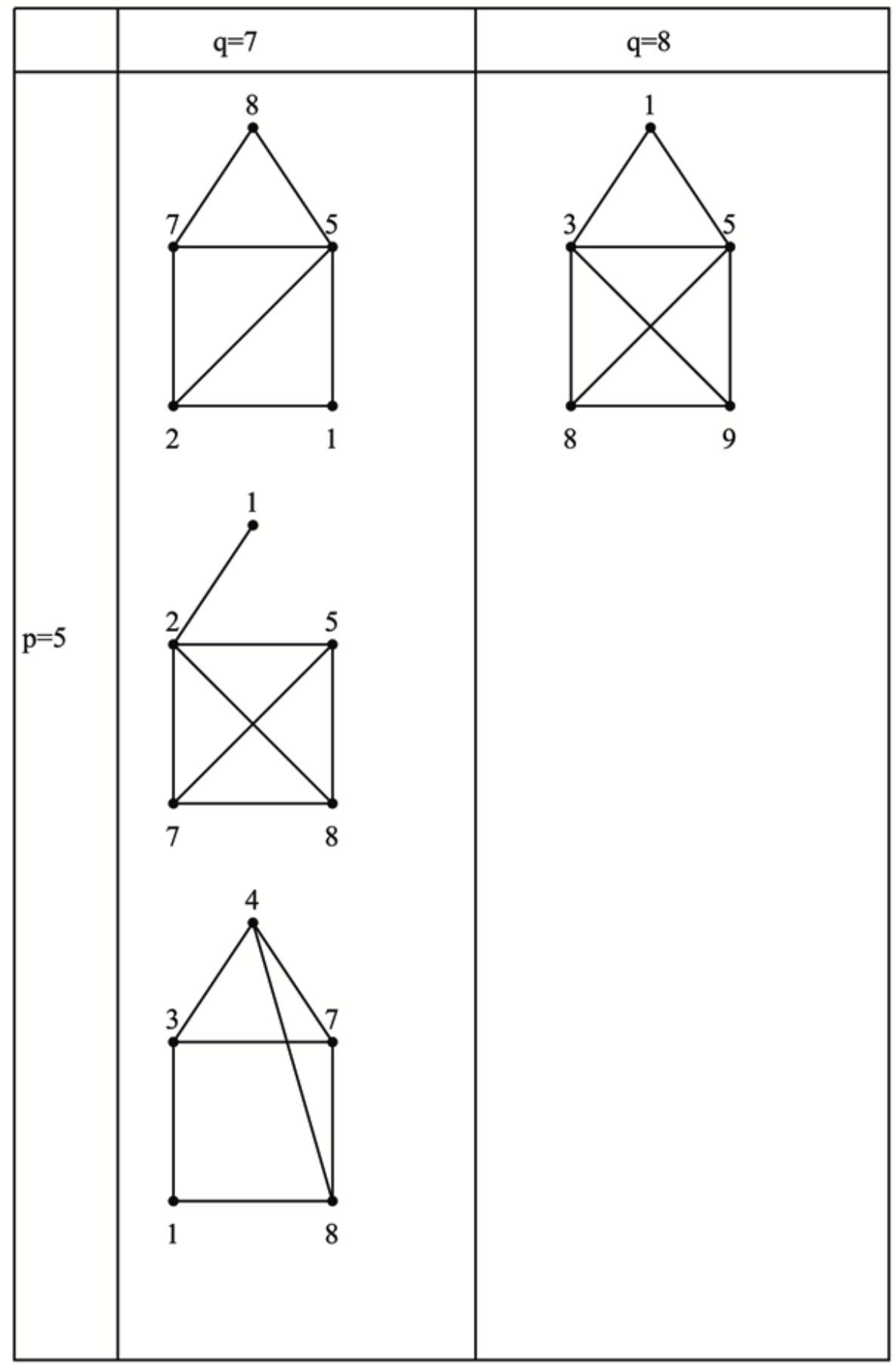

Table 3.3

\begin{tabular}{|c|c|c|}
\hline Order of the graph & Harmonic mean & Not Harmonic mean \\
\hline 1 & 1 & 0 \\
2 & 1 & 1 \\
3 & 2 & 2 \\
4 & 5 & 5 \\
5 & 22 & 13 \\
\hline
\end{tabular}




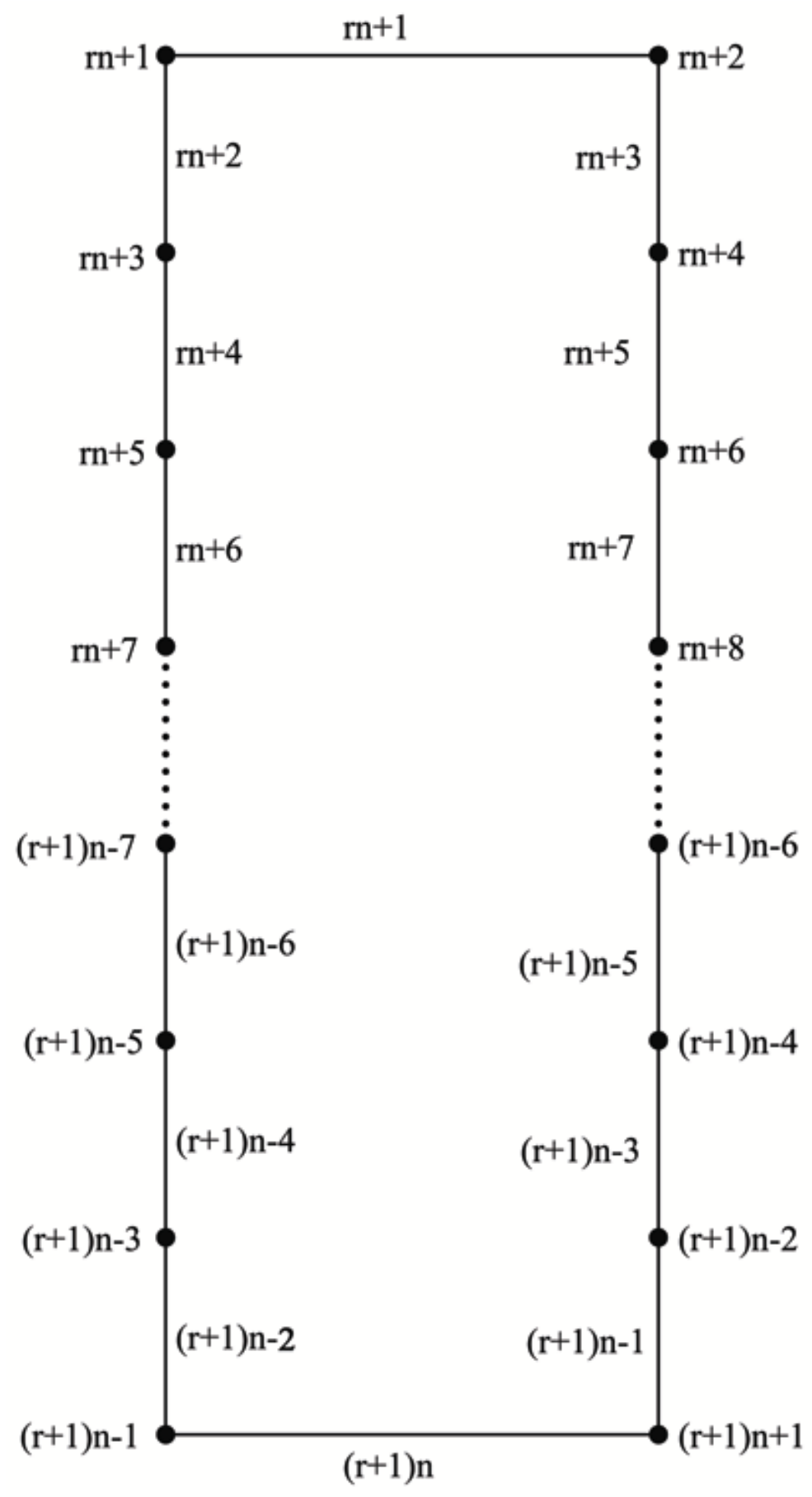

Figure 1 


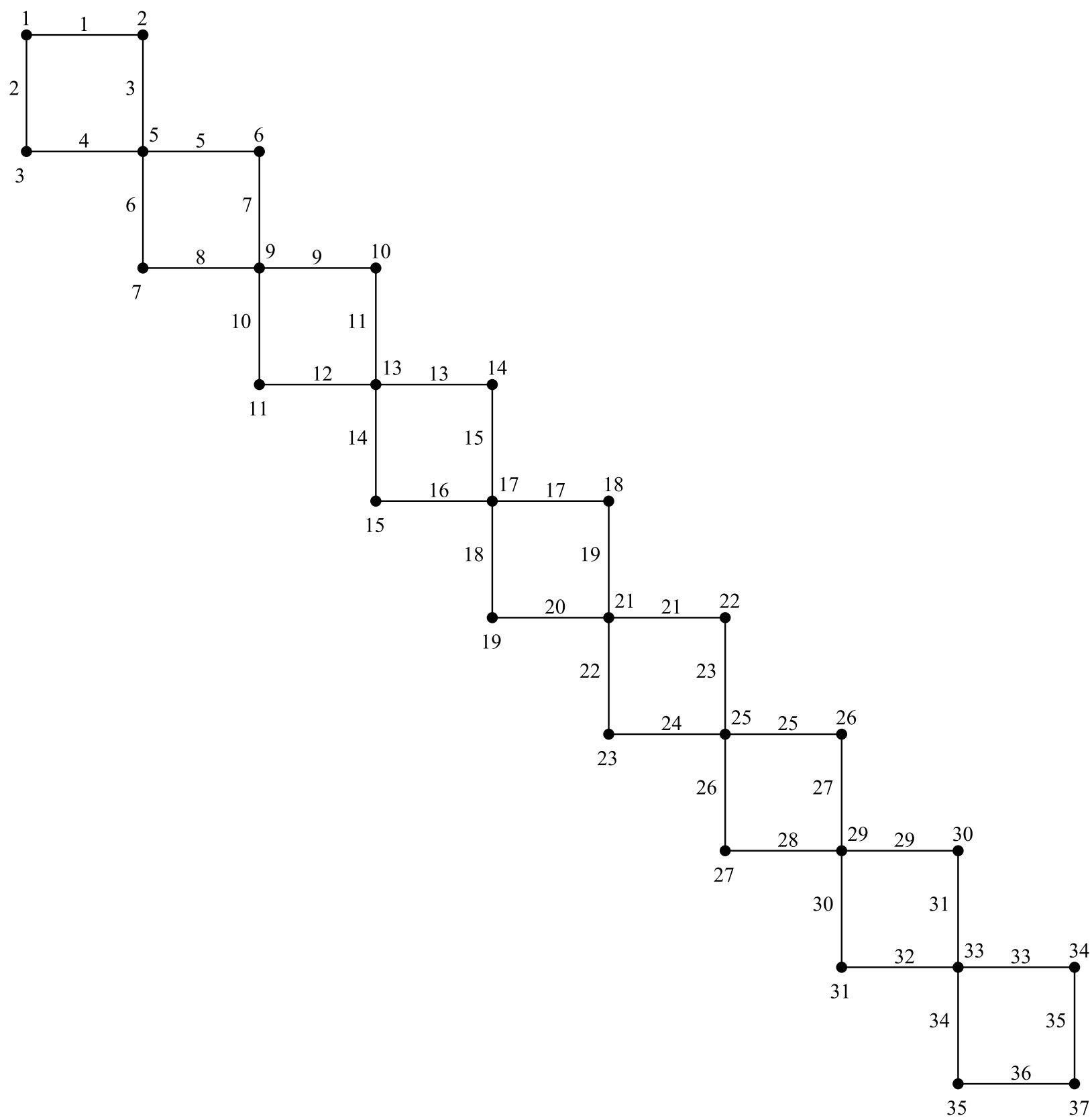

Figure 2

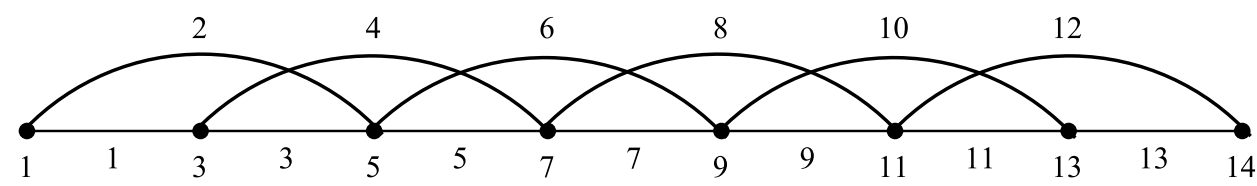

Figure 3 


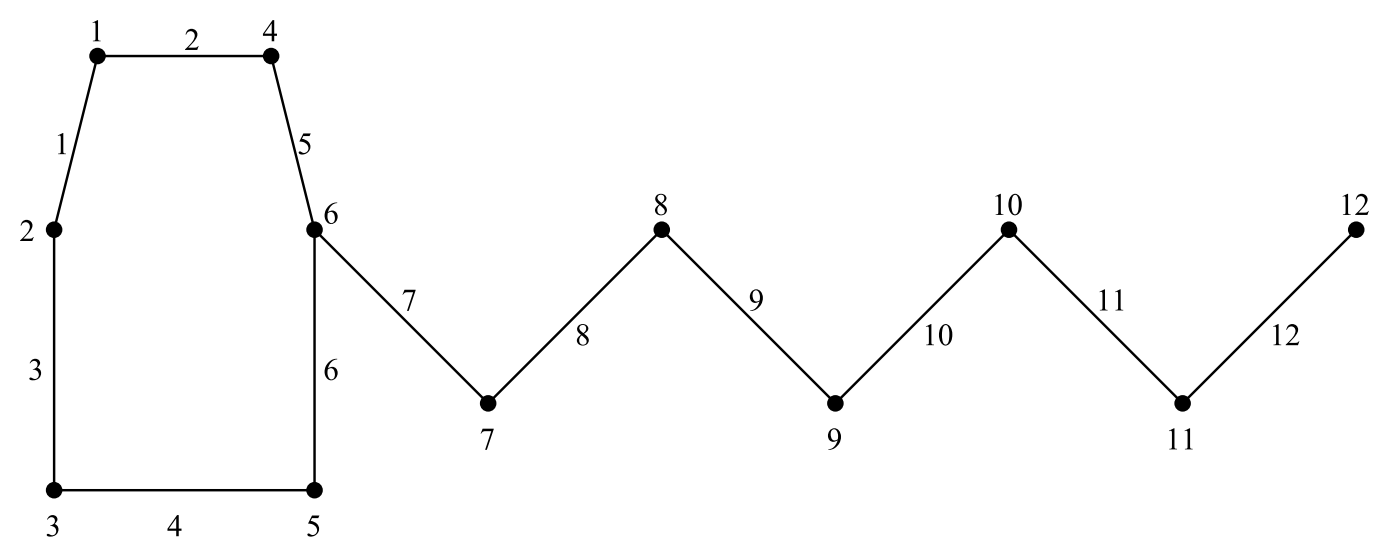

Figure 4
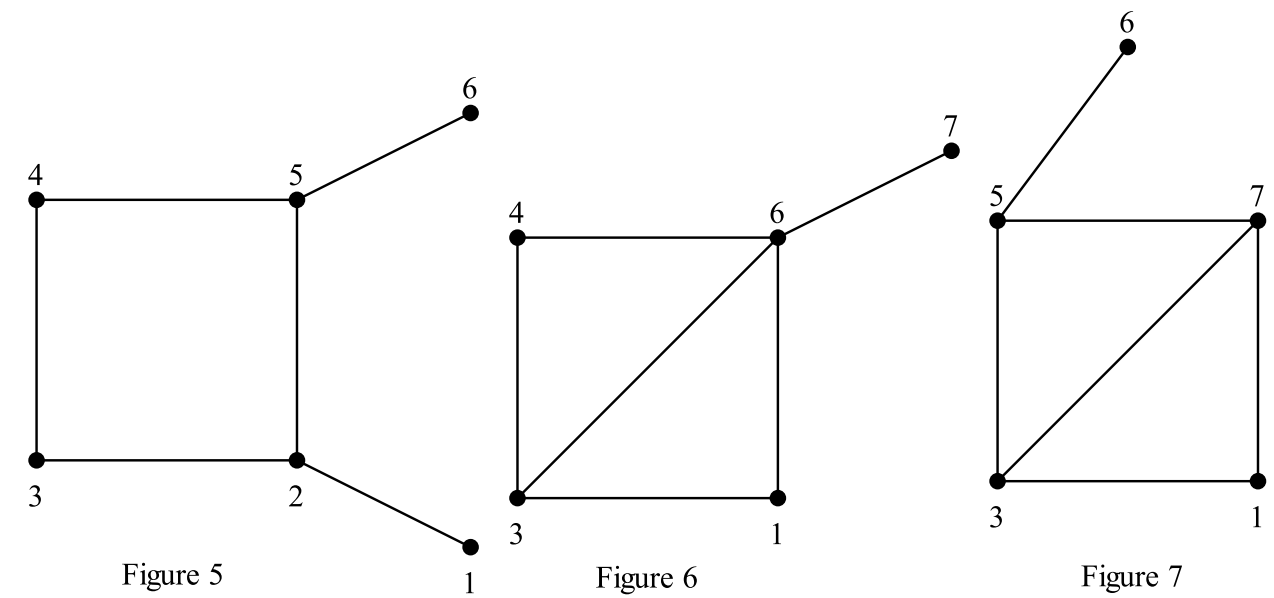

Figure 7 